www.nature.com/pj

\title{
REVIEW
}

\section{Development of self-oscillating polymers and gels with autonomous function}

\author{
Ryo Yoshida ${ }^{1,2}$ \\ In this study, polymers with an autonomous self-oscillating function were examined to obtain a novel biomimetic polymer. \\ The author succeeded in developing a novel self-oscillating polymer and gel by using the oscillating reaction called the \\ Belousov-Zhabotinsky (BZ) reaction, which is recognized as a chemical model for understanding several autonomous phenomena \\ in biological systems. The self-oscillating polymer is composed of a poly $(\boldsymbol{N}$-isopropylacrylamide) network in which the catalyst \\ for the BZ reaction is covalently immobilized. Under the coexistence of reactants, the polymer undergoes spontaneous cyclic \\ soluble-insoluble changes or swelling-deswelling changes (in the case of gel) without any on-off switching of external stimuli. \\ Several kinds of functional material systems using self-oscillating polymer and gel, such as biomimetic actuators and mass \\ transport surfaces, are expected.
}

Polymer Journal (2010) 42, 777-789; doi:10.1038/pj.2010.75; published online 25 August 2010

Keywords: biomimetics; hydrogel; self-oscillation; soft materials

\section{INTRODUCTION}

Many kinds of stimuli-responsive polymer gels that respond to a change in their surroundings, such as changes in temperature, $\mathrm{pH}$ and the electric field, have been developed. They have attracted much attention as smart or biomimetic materials, and several applications, such as actuators (artificial muscle), biosensors, drug delivery systems, purification or separation systems and tissue engineering, have been studied extensively. ${ }^{1-4}$ One of the characteristic behaviors in living systems is autonomous oscillation, that is, spontaneous changes with temporal periodicity (called 'temporal structure'), examples of which are heartbeat, brain waves, pulsatile secretion of hormones, cell cycles and biorhythms. Although several stimuli-responsive polymer systems have been studied from the standpoint of biomimetics, polymer systems undergoing self-oscillation under constant conditions without any on-off switching of external stimuli remain undeveloped. If autonomous polymer systems resembling living organisms can be realized using completely synthetic polymers, unprecedented biomimetic materials may be created.

The author attempted to develop a novel polymer gel that causes autonomous mechanical oscillation without an external control in a completely closed solution. To realize the autonomous polymer system, the Belousov-Zhabotinsky (BZ) reaction, which is well known for exhibiting temporal and spatiotemporal oscillating phenomena, ${ }^{5,6}$ was examined in detail. The $\mathrm{BZ}$ reaction is often analogically compared with the TCA cycle, which is a key metabolic process taking place in the living body, and it is recognized as a chemical model for understanding several autonomous phenomena in biological systems. The overall process of the $\mathrm{BZ}$ reaction is the oxidation of an organic substrate, such as malonic acid (MA) or citric acid, by an oxidizing agent (bromate ion), in the presence of a strong acid and a metal catalyst. In the course of the reaction, the catalyst undergoes spontaneous redox oscillation. When the solution is homogeneously stirred, the color of the solution changes periodically, similar to a neon sign, on the basis of the redox changes of the metal catalyst. When the solution is placed as a thin film in stationary conditions, concentric or spiral wave patterns develop in the solution. The wave of oxidized states propagating in the medium at a constant speed is called a 'chemical wave'. The significance of the BZ reaction has been recognized as a chemical model for understanding some aspects of biological phenomena, such as glycolytic oscillations or biorhythms, cardiac fibrillation, the self-organization of amoeba cells, pattern formation on animal skin and visual pattern processing on the retina.

The author attempted to convert the chemical oscillation of the BZ reaction into a mechanical change in gels and generate an autonomous swelling-deswelling oscillation under non-oscillatory outer conditions. A copolymer gel consisting of $\mathrm{N}$-isopropylacrylamide (NIPAAm) and ruthenium tris $\left(2,2^{\prime}\right.$-bipyridine) ( $\left.\mathrm{Ru}(\mathrm{bpy})_{3}\right)$ was prepared. $\mathrm{Ru}(\mathrm{bpy})_{3}$, functioning as a catalyst for the $\mathrm{BZ}$ reaction, is pendant to the polymer chains of NIPAAm. Poly(NIPAAm) is a wellknown thermosensitive polymer that exhibits a lower critical solution temperature (LCST) of approximately $32^{\circ} \mathrm{C}$, and the homopolymer gel undergoes a volume phase transition at that temperature. For the poly(NIPAAm-co-Ru(bpy $)_{3}$ ) gel, the oxidation of $\mathrm{Ru}(\mathrm{bpy})_{3}{ }^{2+}$ moiety

${ }^{1}$ Department of Materials Engineering, Graduate School of Engineering, The University of Tokyo, Tokyo, Japan and 2PRESTO, Japan Science and Technology Agency (JST), Saitama, Japan

Correspondence: Professor R Yoshida, Department of Materials Engineering, Graduate School of Engineering, The University of Tokyo, 7-3-1 Hongo, Bunkyo-ku, Tokyo, 113-8656, Japan.

E-mail: ryo@cross.t.u-tokyo.ac.jp

Received 27 June 2010; revised 22 July 2010; accepted 23 July 2010; published online 25 August 2010 

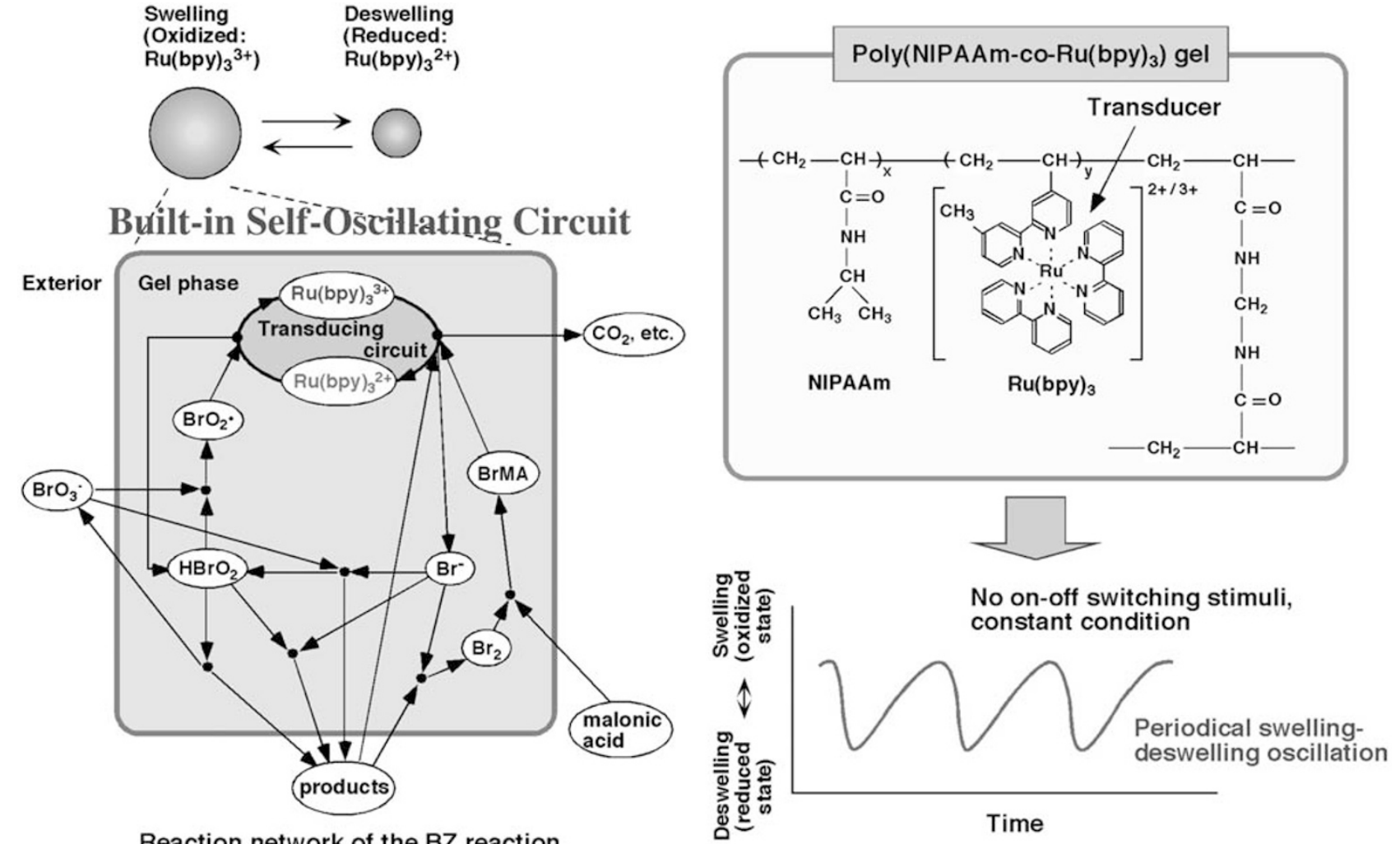

Reaction network of the $\mathrm{BZ}$ reaction (FKN mechanism)

Figure 1 Mechanism of self-oscillation for the poly(NIPAAm-co-Ru(bpy $\left.)_{3}{ }^{2+}\right)$ gel coupled with the Belousov-Zhabotinsky reaction. A full color version of this figure is available at Polymer Journal online.

caused not only an increase in the swelling degree of the gel but also a rise in the volume phase transition temperature. As a result, it is expected that the gel will undergo a cyclic swelling-deswelling change when the $\mathrm{Ru}(\mathrm{bpy})_{3}$ moiety is periodically oxidized and reduced under constant temperature. When the gel is immersed in an aqueous solution containing the substrates of the $\mathrm{BZ}$ reaction (MA, nitric acid and sodium bromate), except for the catalyst, the substrate penetrates the polymer network and the BZ reaction occurs in the gel. Consequently, periodic redox changes induced by the $\mathrm{BZ}$ reaction produce periodic swelling-deswelling changes in the gel (Figure 1).

Since first being reported in 1996 as a 'self-oscillating gel', the author has systematically been studying the self-oscillating polymer and gel, as well as their applications to biomimetic or smart materials (Figure 2). ${ }^{7-46}$ In fact, as applications to autonomic biomimetic actuators, ciliary motion actuators ${ }^{24,25}$ and self-walking gels, ${ }^{26}$ among others, were realized. As an autonomic microconveyor, a mass transport surface was also designed by using the peristaltic motion of the self-oscillating gel. ${ }^{28-30}$ In the case of the uncrosslinked linear polymer, the polymer undergoes spontaneous cyclic solubleinsoluble changes, and the transmittance of the polymer solution oscillates autonomously. ${ }^{32}$ In addition, submicron-sized self-oscillating microgel beads were prepared by a precipitation polymerization method. ${ }^{35-40}$ To realize nanoactuators that exhibit autonomous oscillation on a nanometer scale (nanooscillator), using the linear polymer chain or microgels, their oscillating behavior was investigated through the optical transmittance or viscosity changes ${ }^{39-41}$ of the polymer solution or microgel dispersions.

\section{SELF-OSCILLATING BEHAVIOR OF THE GEL}

Self-oscillation of gels with smaller size compared with chemical wavelength and control of oscillating behavior

Figure 3 shows the observed oscillating behavior under a microscope for the small cubic poly(NIPAAm-co-Ru(bpy) ${ }_{3}{ }^{2+}$ ) gel (each has a length of about $0.5 \mathrm{~mm}$ ). In miniature gels that are sufficiently smaller than the wavelength of the chemical wave (typically several $\mathrm{mm}$ ), the redox change of the ruthenium catalyst can be regarded to occur homogeneously without pattern formation. ${ }^{12}$ Because of the redox oscillation of the immobilized $\mathrm{Ru}(\mathrm{bpy})_{3}{ }^{2+}$, the mechanical swellingdeswelling oscillation of the gel autonomously occurs with the same period found for the redox oscillation. The volume change is isotropic, and the gel beats as a whole, similar to a heart muscle cell. The chemical and mechanical oscillations are synchronized without a phase difference (that is, the gel exhibits swelling during the oxidized state and deswelling during the reduced state).

Typically, the oscillation period increases with a decrease in the initial concentration of substrates. Further, in general, the oscillation frequency (the reciprocal of the period) of the BZ reaction tends to increase as the temperature increases, in accordance with the Arrhenius equation. The swelling-deswelling amplitude of the gel increases with an increase in the oscillation period and amplitude of the redox changes. Therefore, the swelling-deswelling amplitude of the gel is controllable by changing the initial concentration of substrates, as well as temperature.

An inherent behavior of the $\mathrm{BZ}$ reaction, the abrupt transition from the steady state (non-oscillating state) to the oscillating state, occurs with a change in a controlling parameter, such as chemical composition 
Polymer chain

$$
\begin{gathered}
\text { Oscillation } \\
\begin{array}{c}
\text { Soluble } \\
\text { (Oxidized state) }
\end{array} \\
\begin{array}{c}
\text { Insoluble } \\
\text { (reduced state) }
\end{array}
\end{gathered}
$$

Self-oscillation of polymer chains
- Transmittance oscillation

- Viscosity oscillation

Self-flocculating/ dispersing oscillation of microgels

\section{Self-oscillating gel}
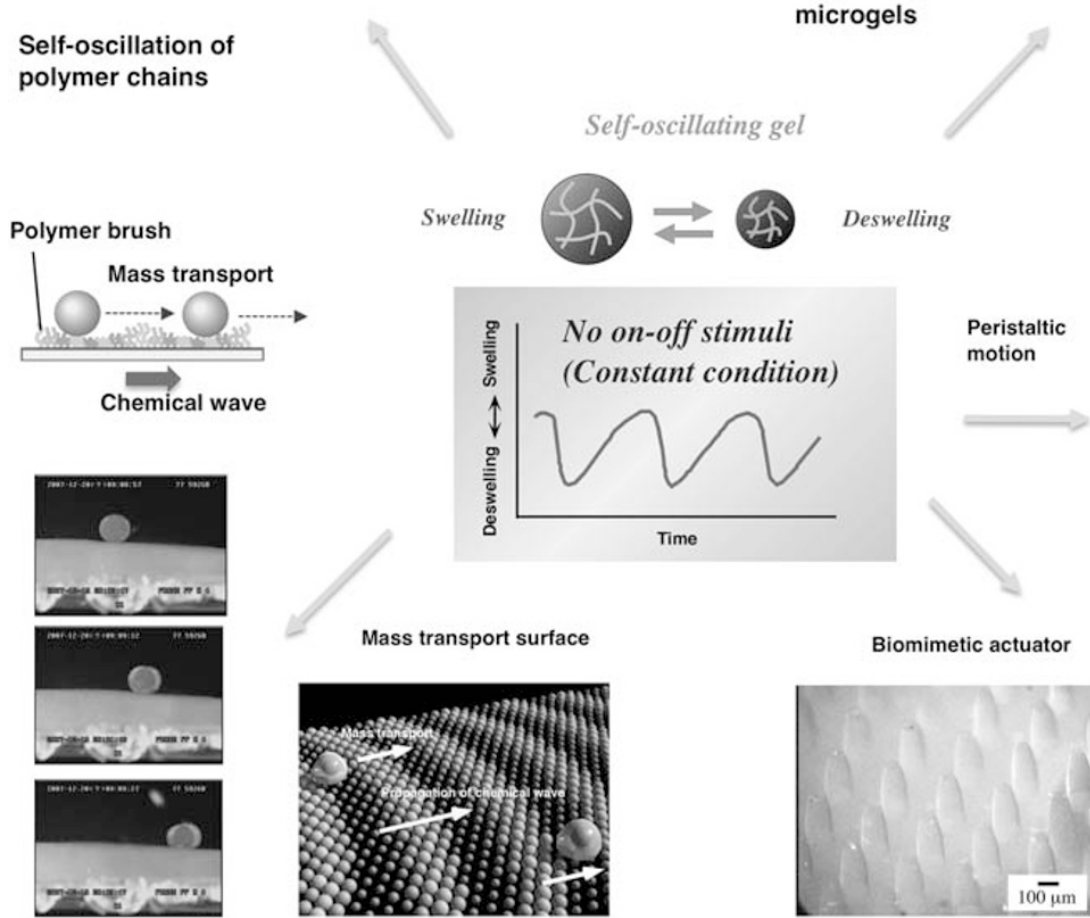

Mass transport surface

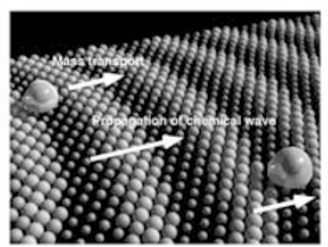

Biomimetic actuator

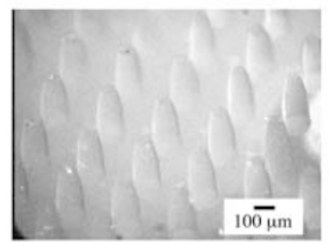

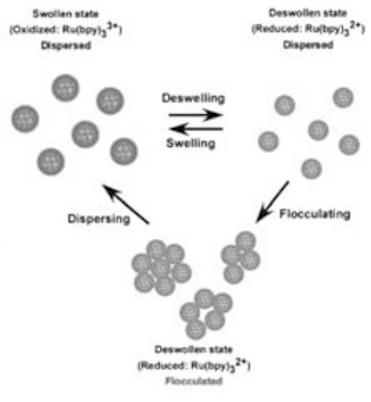

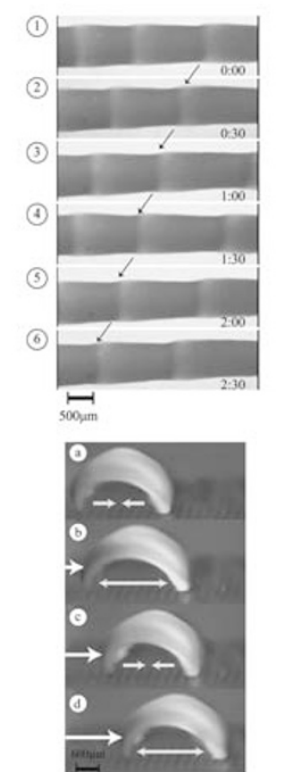

Figure 2 Development of self-oscillating polymers and gels. A full color version of this figure is available at Polymer Journal online.

or light (Figure 4). By using these characteristics, the reversible on-off regulation of self-beating triggered by the addition and removal of MA was successfully achieved. ${ }^{13}$ We showed the oscillating behavior of the gel when the stepwise concentration change in MA was repeated between a lower concentration $(10 \mathrm{~mm})$ in the steady state and a higher concentration $(25 \mathrm{~mm})$ in the oscillating state. At $10 \mathrm{~mm}$, redox oscillation did not occur; consequently, the gel exhibited no swellingdeswelling changes. Thereafter, the concentration was quickly increased to $25 \mathrm{~mm}$. Immediately after increasing the concentration, the gel began self-beating. The beating stopped again as soon as the concentration was reduced to the initial value. In this way, the reversible on-off regulation of self-beating triggered by MA was successfully achieved. As there are some organic acids that can be the substrate for the $\mathrm{BZ}$ reaction (for example, citric acid), the same regulation of beating is possible using these organic acids instead of MA. As the gel has thermosensitivity because of the NIPAAm component, the beating rhythm can also be controlled by temperature. By using this thermosensitivity, the on-off control of the self-oscillation is also possible. At lower temperatures, the oscillation does not occur because there is no remarkable difference in the hydrophilicity of the polymer between the redox states, but the difference becomes remarkable at higher temperatures and causes self-oscillation (Figure $4 \mathrm{~b}) .{ }^{14}$

The period of oscillation is affected by light illumination for the $\mathrm{Ru}(\text { bpy })_{3}{ }^{2+}$-catalyzed BZ reaction. Therefore, we can intentionally make a pacemaker with a desired period (or wavelength) by local illumination of the gel with a laser beam, or we can change the period (or wavelength) by the local illumination of a pacemaker that already exists in the gel. ${ }^{15}$ The optical on-off control of the self-oscillating motion of the gel was demonstrated (Figure $4 \mathrm{c}$ ). ${ }^{16-18}$

\section{Peristaltic motion of gels with propagation of chemical wave}

When the gel size is larger than the chemical wavelength, the chemical wave propagates in the gel by coupling with the diffusion of intermediates. Thereafter, a peristaltic motion of the gel is created. Figure 5A shows the cylindrical gel, which is immersed in an aqueous solution containing the three reactants of the BZ reaction. The chemical waves propagate in the gel at a constant speed in the direction of the gel length. ${ }^{19}$ Considering that the orange ( $\left.\mathrm{Ru}(\mathrm{II})\right)$ and green $(\mathrm{Ru}(\mathrm{III}))$ zones simply represent the shrunken and swollen parts, respectively, the locally swollen and shrunken parts move with the chemical wave, similar to the peristaltic motion of living worms.

The tensile force of the cylindrical poly(NIPAAm-Co-Ru(bpy $)_{3}{ }^{2+}$ ) gel with oscillation was also measured. ${ }^{20}$ Further, the swelling behavior of the gel during self-oscillation was observed using a CCD camera, and the force due to the $\mathrm{BZ}$ reaction was evaluated using a semiconductor gauge. ${ }^{21}$ Self-oscillating swelling-deswelling behavior in samples with different swelling ratios was evaluated by this apparatus, and mechanical parameters such as Young's modulus, stress amplitude and oscillating period were quantitatively estimated. The dependency of the initial length in samples on Young's modulus seems to be related to the propagation of the chemical wave in the samples. 

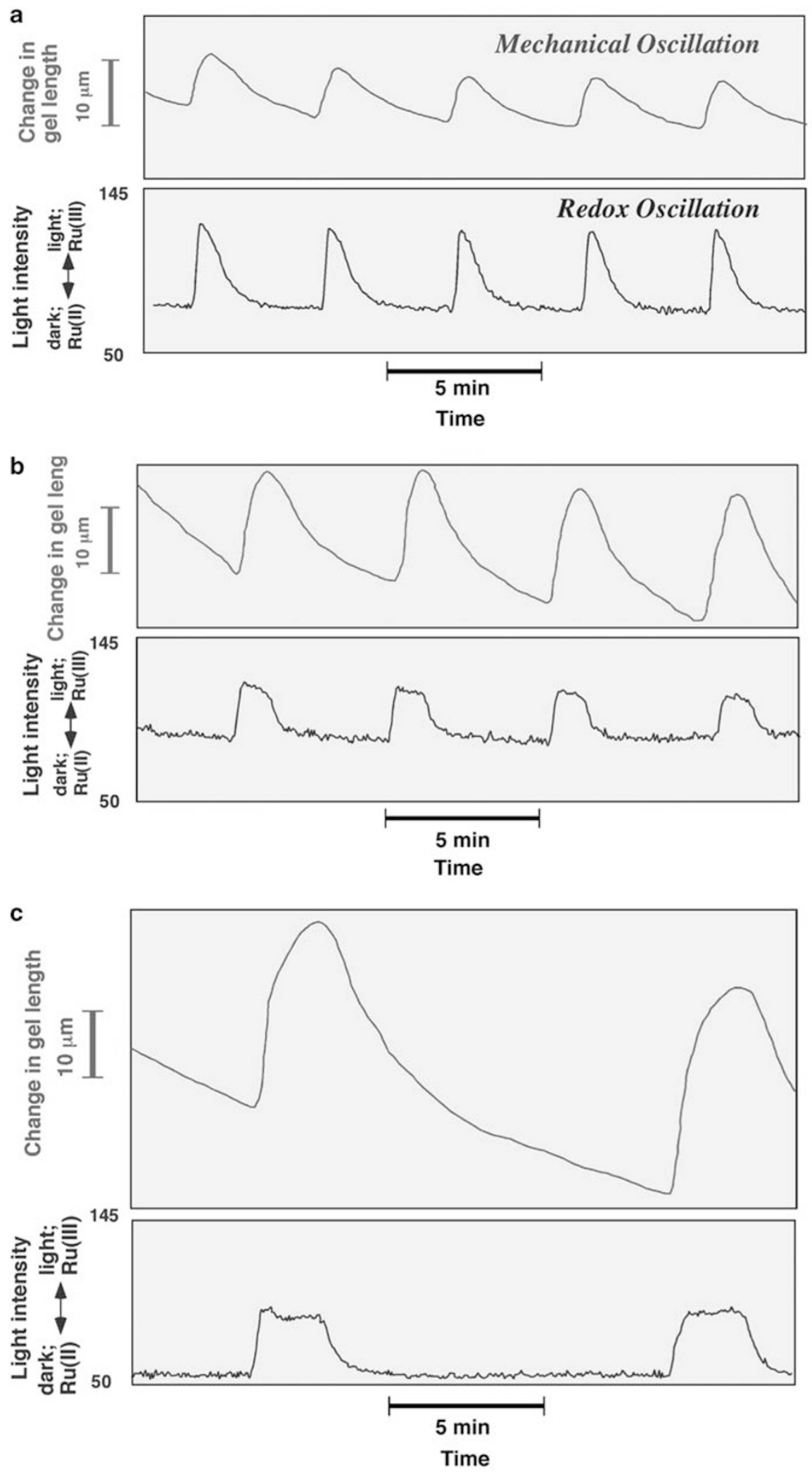

Figure 3 Periodic redox changes of the miniature cubic poly(NIPAAm-co-Ru(bpy) ${ }_{3}{ }^{2+}$ ) gel (lower) and the swelling-deswelling oscillation (upper) at $20^{\circ} \mathrm{C}$. Color changes in the gel accompanied by redox oscillations (orange: reduced state; light green: oxidized state) were converted into 8-bit grayscale changes (dark: reduced; light: oxidized) by image processing. Transmitted light intensity is expressed as an 8-bit grayscale value. Outer solution: [MA] $=62.5 \mathrm{~mm}$; $\left[\mathrm{NaBrO}_{3}\right]=84 \mathrm{mM} ;\left[\mathrm{HNO}_{3}\right]=(\mathbf{a}) 0.6 \mathrm{~m},(\mathbf{b}) 0.675 \mathrm{~m}$ and (c) $0.894 \mathrm{~m}$ (Yoshida et al. ${ }^{12}$ ). A full color version of this figure is available at Polymer Journal online. 


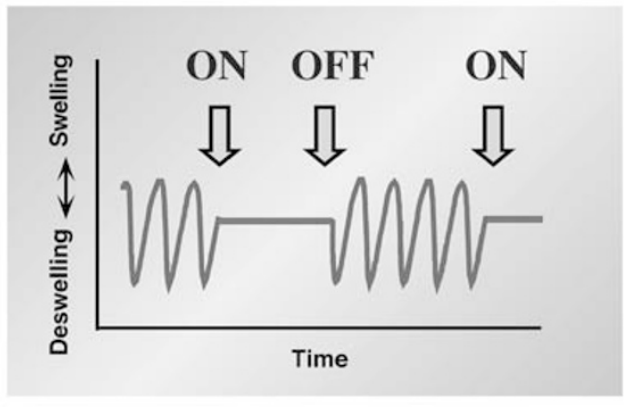

a
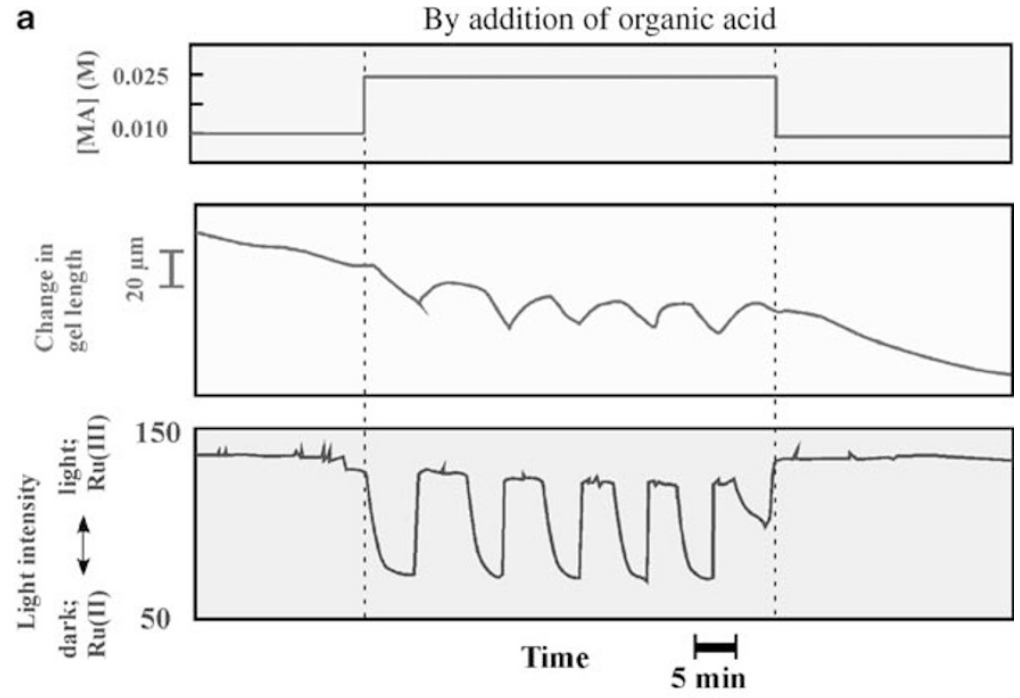

b

By temperature

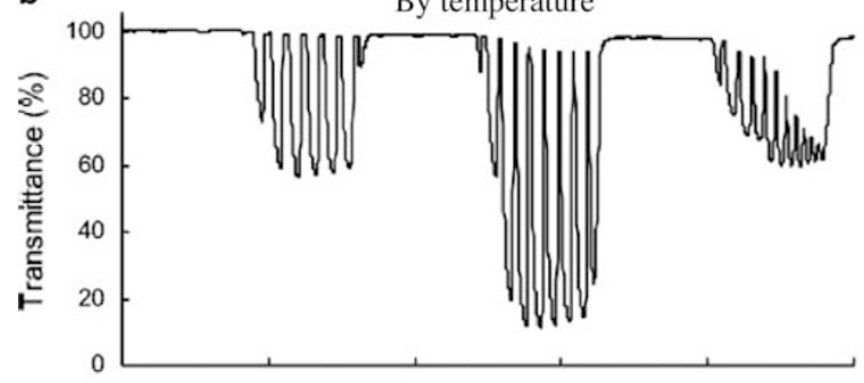

By photo-irradiation

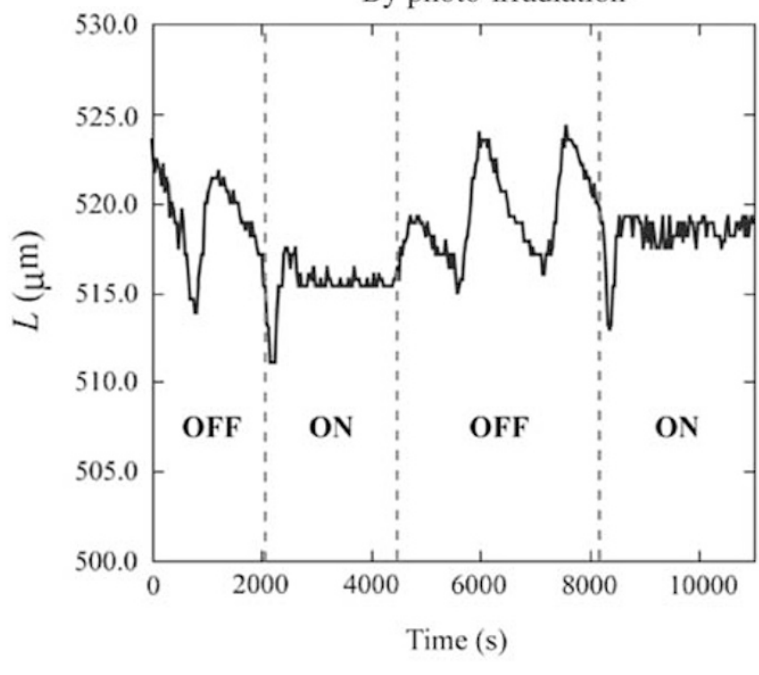

Figure 4 (a) Change in the oscillating behavior of the gel in response to the stepwise concentration changes of MA between 10 and $25 \mathrm{mM}$ (others: $\left.\left[\mathrm{NaBrO}_{3}\right]=84 \mathrm{~mm},\left[\mathrm{HNO}_{3}\right]=0.3 \mathrm{~m}, 20^{\circ} \mathrm{C}\right)\left(\right.$ Yoshida et al. $\left.{ }^{13}\right)$. (b) Oscillating profiles of optical transmittance for poly(NIPAAm-co-Ru(bpy) ${ }_{3}^{2+}$-co-AAc) solution $\left([\mathrm{MA}]=0.1 \mathrm{M},\left[\mathrm{NaBrO}_{3}\right]=0.2 \mathrm{M},\left[\mathrm{HNO}_{3}\right]=0.3 \mathrm{~m}\right)$ under the condition that temperature was changed stepwise (Ito et al. ${ }^{14}$ ). (c) On-off switching of the peristaltic motion by photoirradiation. While the light-irradiated state (ON state) and the dark state (OFF state) were alternated, the thickness of the gel was measured. Light intensity was $6.45 \mathrm{~mW}$. The initial compositions of the reaction solution were $\left[\mathrm{NaBrO}_{3}\right]=0.028 \mathrm{M},[\mathrm{MA}]=0.0625 \mathrm{M}$ and $\left[\mathrm{HNO} \mathrm{O}_{3}\right]=0.890 \mathrm{M}$ (Shinohara et al. ${ }^{18}$ ). A full color version of this figure is available at Polymer Journal online.

\section{DESIGN OF BIOMIMETIC ACTUATOR USING SELF-OSCILLATING POLYMER AND GEL}

Microfabrication of self-oscillating gel for microdevices

Recently, microfabrication technologies such as photolithography have also been attempted for the preparation of microgels. As any shape of gel can be created by these methods, application as a new manufacturing method for soft microactuators, microgel valves and gel displays, among others, is expected. The microfabrication of gels has also been attempted by photolithography for application to such microdevices. ${ }^{22,23}$ One possible application using the self-oscillating gel may be a self-oscillatory drug release microchip. The use of a microfabricated self-oscillating gel as a beating micropump to push and pull a diaphragm that separates a drug reservoir in a microchip ${ }^{22}$ can represent a microchip to release a drug periodically. As the selfbeating of the gel occurs in the closed solution containing BZ reactants as an energy source, a complete stand-alone microchip without electric wiring and external apparatus is possible. The periodic release of a drug with preprogrammed periods under constant conditions can be achieved, and it would lead to several effects, such as applications to chronopharmacotherapy to release hormones synchronized with biorhythms and decreasing drug tolerance.

\section{Ciliary motion actuator using self-oscillating gel}

One of the promising fields of the MEMS is the microactuator array or distributed actuator systems. The actuators, which have a very simple actuation motion, such as an up and down motion, are arranged in an array. If their motions are random, no work is extracted from this array. However, by controlling them to operate in a certain order, they can generate work as a system. A typical example of this kind of actuation array is a ciliary motion microactuator array. There have 


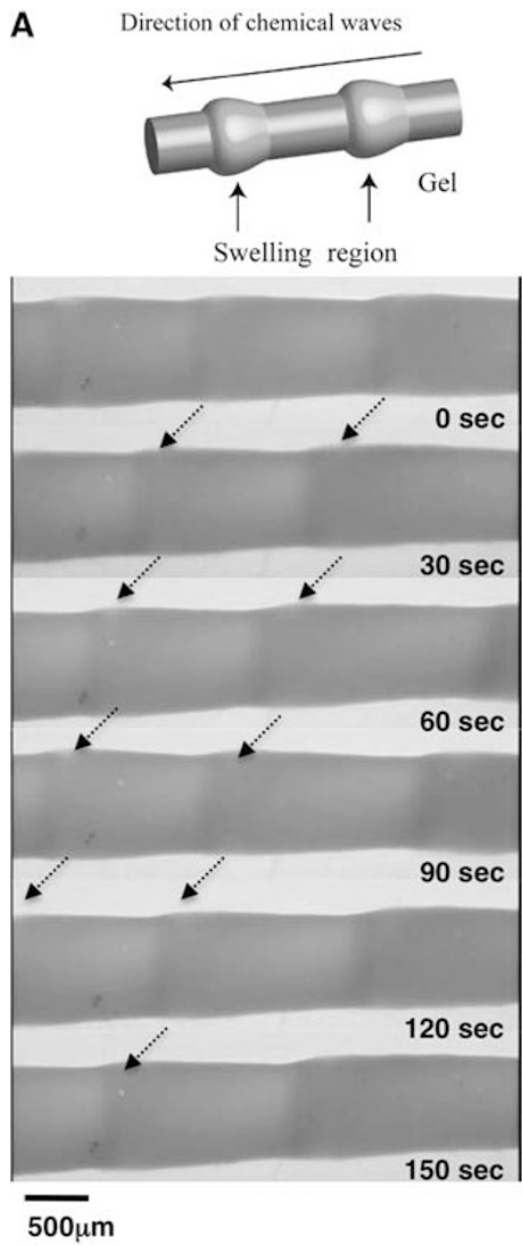

B
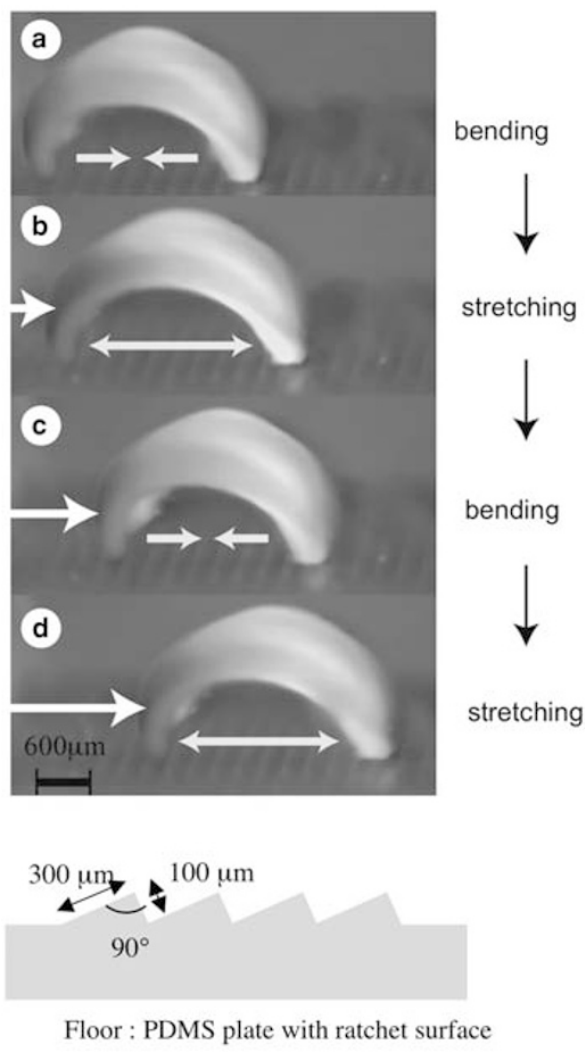

Figure 5 (A) Time course of the peristaltic motion of the poly(NIPAAm-co-Ru(bpy) ${ }^{2+}$-co-AMPS) gel in a solution of BZ substrates at $18{ }^{\circ} \mathrm{C}$. The green and orange colors correspond to the oxidized and reduced states of the Ru moiety in the gel, respectively (Maeda et al. ${ }^{19}$ ). (B) Time course of the self-walking motion of the gel actuator. During stretching, the front edge can slide forward on the base, but the rear edge is prevented from sliding backward. On the contrary, during bending, the front edge is prevented from sliding backward, whereas the rear edge can slide forward. This action is repeated, and as a result, the gel moves forward. Outer solution: $[\mathrm{MA}]=62.5 \mathrm{~mm},\left[\mathrm{NaBrO}_{3}\right]=84 \mathrm{~mm},\left[\mathrm{HNO}_{3}\right]=0.894 \mathrm{~m}, 18^{\circ} \mathrm{C}$ (Maeda et al. ${ }^{26}$ ). A full color version of this figure is available at Polymer Journal online.

been many reports on this system. Although various actuation principles have been proposed, all the previous works are based on the concept that the motion of actuators is controlled by external signals. If a self-oscillating gel plate with a microprojection structure array on top was realized, it would be expected that the chemical wave propagation would create a dynamic rhythmic motion of the structure array. This proposed structure could exhibit spontaneous dynamic propagating oscillation, producing a ciliary motion array. ${ }^{24,25}$

A gel plate with a microprojection array was fabricated by molding. First, moving mask deep X-ray lithography was used to fabricate a PMMA plate with a truncated conical shape microstructure array. This step was followed by the evaporation of an Au seed layer and the subsequent electroplating of nickel to form the metal mold structure. Thereafter, a PDMS mold structure was duplicated from the $\mathrm{Ni}$ structure and used for gel molding. The formation of gel was carried out by vacuum injection molding. A structure with a height of $300 \mu \mathrm{m}$ and bottom diameter of $100 \mu \mathrm{m}$ was successfully fabricated by the described process. The propagation of the chemical reaction wave and dynamic rhythmic motion of the microprojection array were confirmed by observing the chemical wave and performing displacement measurements. The motion of the top was observed as having a $5 \mu \mathrm{m}$ range in both lateral and vertical directions and an elliptical motion of the projection top. The feasibility of the new concept of the ciliary motion actuator made of self-oscillating polymer gel was successfully confirmed. The actuator may serve as a microconveyer to transport micro- or nanoparticles on the surface.

\section{Self-walking gel}

Further, we successfully developed a novel biomimetic walking-gel actuator made of self-oscillating gel. ${ }^{26}$ To produce a directional movement of the gel, asymmetrical swelling-deswelling is desired. For these purposes, as a third component, hydrophilic 2-acrylamido2'-methylpropanesulfonic acid (AMPS) was copolymerized into the polymer to lubricate the gel and cause anisotropic contraction. During polymerization, the monomer solution faces two different surfaces of plates: a hydrophilic glass surface and a hydrophobic Teflon surface. As the thickness of the spacer is thin $(0.5 \mathrm{~mm})$, the surface property of the plate may affect the distribution of the monomer in the solution. As the $\mathrm{Ru}(\mathrm{bpy}){ }_{3}{ }^{2+}$ monomer is hydrophobic, it easily migrates to the side of the Teflon surface. As a result, a non-uniform distribution along the height is formed by the components, and the resulting gel has a gradient distribution for the content of each component in the polymer network. 
To convert the bending and stretching changes to one-directional motion, we used a ratchet mechanism. A ratchet base with an asymmetrical surface structure was fabricated. On the ratchet base, the gel repeatedly bends and stretches autonomously, resulting in a forward motion of the gel, whereas sliding backward is prevented by the teeth of the ratchet. Figure 5B shows successive profiles of the 'self-walking' motion of the gel similar to a looper in the BZ substrate solution under constant temperature. The walking velocity of the gel actuator was $\sim 170 \mu \mathrm{mmin}^{-1}$. As the oscillating period and the propagating velocity of the chemical wave change with the concentration of substrates in the outer solution, the walking velocity of the gel can be controlled. Using the gel with a gradient structure, another type of actuator, one that generates a pendulum motion, is also realized. ${ }^{27}$

Mass transport surface using the peristaltic motion of the gel Further, we attempted to transport an object by using the peristaltic motion of the self-oscillating gel. ${ }^{28-30}$ To control transportability, it is necessary to enhance the driving force as a conveyer. It is effective to copolymerize AMPS to the poly(NIPAAm-co-Ru(bpy $)_{3}{ }^{2+}$ ) gel network to generate a large amplitude change in the volume of the selfoscillating gel. For the design of a mass transport surface, the influence of the AMPS's feed ratio on the swelling-deswelling properties of poly(NIPAAm-co-Ru(bpy) ${ }_{3}{ }^{2+}$-co-AMPS) gels and the mass transportability of gels were investigated.

The gel had a microphase-separated structure when the AMPS's feed ratio was $<5 \mathrm{~mol} \%$ because of the effect of the poor solvent on the polymerization process. However, when the AMPS's feed ratio was more than $10 \mathrm{~mol} \%$, the gel had a homogeneous structure. The microphase-separated structure highly improved the swelling-deswelling kinetics and generated a swelling-deswelling amplitude of $>10 \%$ of the gel's thickness, which was $\sim 10$ times larger than that of the gel with a homogeneous network structure.

As a model object, a cylindrical poly(acrylamide) (PAAm) gel was placed on the gel surface. The PAAm gel was transported onto the gel surface with the propagation of the chemical wave, as it rolled (Figure 6a) when the AMPS's feed ratio was low ( $<2.5 \mathrm{~mol} \%)$. Figure $6 \mathrm{~b}$ shows a phase diagram of transportable conditions given by the velocity and inclination angle $\theta$ of the wave front. It was found that the cylindrical PAAm gel was not transported if the inclination angle was less than $\sim 0.05$ rad. It seems that the mass transportability depends not on the velocity of the chemical wave but rather on the diameter of the cylindrical PAAm gel and the inclination angle of the wave front. We have proposed a model to describe the mass transport phenomena based on the Hertz contact theory, and the relation between transportability and peristaltic motion was investigated.

Figure $6 \mathrm{c}$ shows the contact model of the cylindrical PAAm gel and the poly(NIPAAm-co-Ru(bpy) ${ }_{3}{ }^{2+}$-co-AMPS) gel sheet. If the inclination angle of the poly(NIPAAm-co-Ru(bpy) $3^{2+}$-co-AMPS) gel surface is smaller than the slope of the tangent at point A, sufficient contact force from the strain does function to rotate the cylindrical PAAm gel. Therefore, the condition for the transport of the cylindrical PAAm gel is written as $R_{C} \sin \theta \geqq b$.

The minimum inclination angle calculated from the above equation was the same as the angle resulting from the experiment. It was found experimentally and supported by the model that the sheer wave front of the peristaltic motion was necessary to transport cylindrical gels. As the size of the gels ranged approximately from sub-mm to $\mathrm{mm}$ order, gravity, frictional force and contact force acting on the PAAm gel were the important factors driving mass transport. Therefore, if the cargo materials are not adsorbed to the poly(NIPAAm-co-Ru(bpy) ${ }_{3}{ }^{2+}$ co-AMPS) gel surface by an attractive force such as hydrophobic a
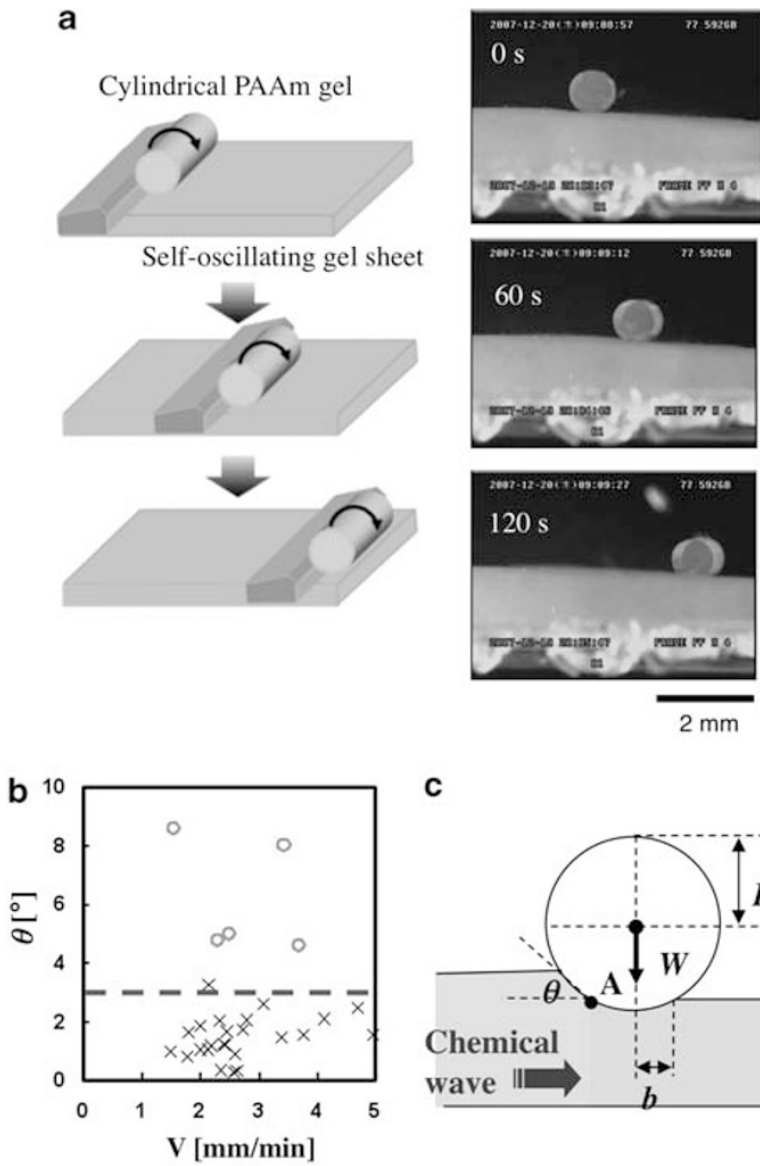

C

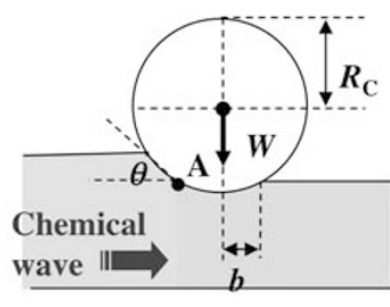

Figure 6 (a) Schematic illustration of mass transport on the peristaltic surface (left) and the observed transport of cylindrical PAAm gel on the poly(NIPAAm-co-Ru(bpy) $3^{2+}$-co-AMPS) gel sheet (right). (b) Phase diagram of the transportable region given by the velocity and inclination angle of the wave front: $(O)$ transported, $(x)$ not transported. (c) Model of the rolling cylindrical gel on the peristaltic gel surface $\left(R_{\mathrm{C}}=\right.$ radius of curvature, $W=$ load of the PAAm gel, $b=$ contact half-width) (Murase et al. ${ }^{28}$ ). A full color version of this figure is available at Polymer Journal online.

interaction, mass transportation depends on the performance of the peristaltic motion of the poly(NIPAAm-co-Ru(bpy) ${ }_{3}{ }^{2+}$-co-AMPS) gel surface. It was found that the microphase-separated structure is a key to transport cylindrical gels because the enlarged peristaltic motion could be useful for generating the rotational movement of the cylindrical gel.

Further, the surface figure capable of transporting microparticles in one direction was designed to fabricate a more versatile self-driven gel conveyer. The self-oscillating gel having a grooved surface was fabricated, and the effectiveness of the surface design was investigated. Poly(AAm-co-AMPS) gel beads with diameters of several hundred $\mu \mathrm{m}$ to several $\mathrm{mm}$ were transported onto the grooved surface of the selfoscillating gel by its autonomous peristaltic motions (Figure 7A) ${ }^{30}$ It was found that the traveling direction of the peristaltic motion could be confined to the direction along the grooves by designing the distance between the grooves to be shorter than the wavelength of the chemical wave. Consequently, several gel beads were transported in parallel. The contact areas of loaded cargos and the self-oscillating gel surface were calculated theoretically to investigate the relationship between transportability and the surface figure.

We tried to estimate the contact area in each experimental case in which loaded gel cargos were gel bead and cylindrical gel. ${ }^{30}$ The 
A

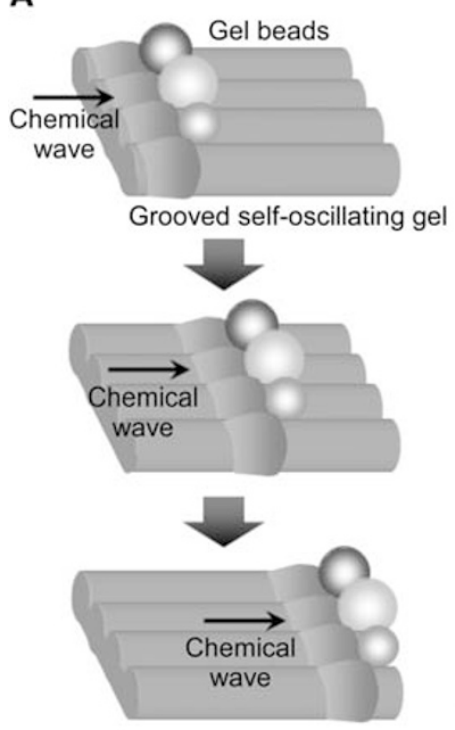

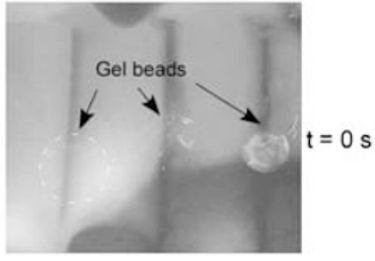

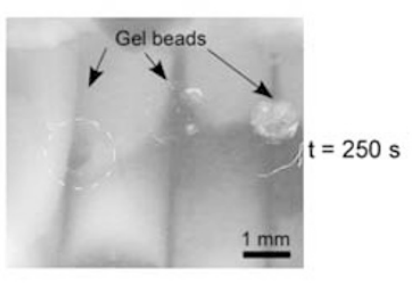

B

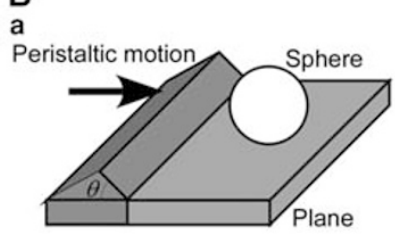

b Peristaltic motion Sphere

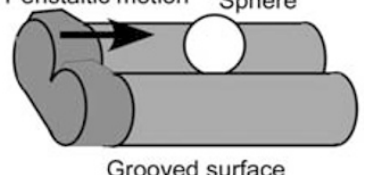

Grooved surface

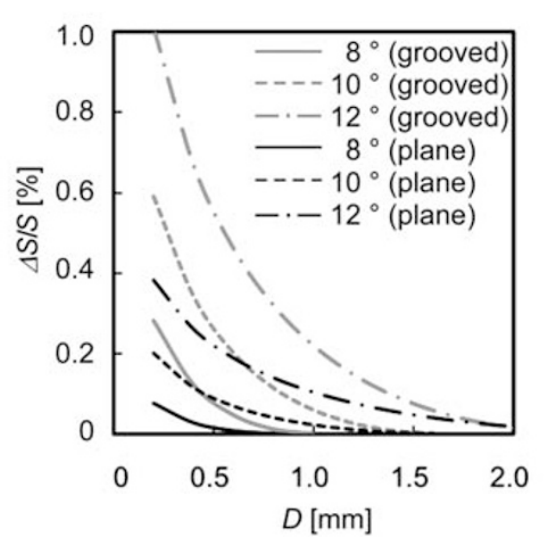

Figure 7 (A) Schematic illustration of mass transport on the peristaltic surface (left) and transport of poly(AAm-co-AMPS) gel beads on the grooved surface of the self-oscillating gel. Outer solution: $[\mathrm{MA}]=62.5 \mathrm{~mm},\left[\mathrm{NaBrO}_{3}\right]=84 \mathrm{~mm},\left[\mathrm{HNO}_{3}\right]=0.894 \mathrm{~m}, 18^{\circ} \mathrm{C}$ (Murase et al. ${ }^{30}$ ). (B) (Upper) Schematic illustrations of a loaded cargo and a self-oscillating gel surface; (A) a sphere on a flat surface, (B) a sphere on a groove between two adjacent cylinders. (Lower) Dependence of the increase in the contact area during peristaltic motion on the diameter of the sphere and the inclination angle of the wave front. A sphere is loaded on the groove (red line) or on the plane (black line). The inclination angles of the wave front used in the calculation are 8,10 and $12^{\circ}$, respectively. A full color version of this figure is available at Polymer Journal online.

contact areas between (a) a sphere and a flat surface, (b) a sphere and a grooved surface, (c) a cylinder and a flat surface and (d) a cylinder and a grooved surface can be calculated theoretically from the Hertz contact theory. As the self-oscillating gel exhibits peristaltic motions, two situations were considered. One was the case that the loaded sphere or cylinder is just put on the surface during rest, and the other was the case that the wave front of the peristaltic motion reaches the loaded cargo. By calculating a contact area in each situation, we clarified the increase in contact area $(\Delta S)$ by the peristaltic motion.

The calculated $\Delta S$ for the sphere ((a) a sphere and a flat surface, (b) a sphere and a grooved surface) was summarized in Figure $7 \mathrm{~B}$ as a function of the diameter. The increase in contact area was normalized by the surface area. The red line shows the case in which the sphere is on the grooved surface, and the black line shows the case in which the sphere is on the flat surface. The inclination angle was set as 8,10 or $12^{\circ}$ in both cases. Figure 7B shows that the increase of the contact area during the peristaltic motion increases when the inclination of the wave front becomes sheerer, and the increase of the contact area on the grooved surface is larger than that on the flat surface. The $x$-intercept of the calculated line represents the maximum transportable diameter of the sphere. Through transport experiments, the inclination angle of the wave front on the flat gel surface was, on an average, $5.3^{\circ}$. In contrast, the inclination angle on the grooved gel surface was, on an average, $10^{\circ}$. The diameter of the gel bead used in the experiment ranged from around sub-mm to several $\mathrm{mm}$. Therefore, it can be estimated that $\Delta S / S$ of the sphere with a diameter of sub-mm to $\mathrm{mm}$ on the flat surface $\left(\theta=5.3^{\circ}\right)$ is nearly $0 \%$ and that the inclination angle of the wave front on the flat surface is not sheer enough to transport spheres. On the other hand, $\Delta S / S$ of the sphere on the grooved surface $\left(\theta=10^{\circ}\right)$ is not $0 \%$, and the sphere can be transported. It was found that the surface design to form grooves was efficient for increasing the contact area when the wave front of the peristaltic motion reached the loaded cargo, and was useful for transporting not only cylindrical cargos but also spherical cargos in desired directions.

The functional gel surface generating an autonomous and periodic peristaltic motion has a potential for several new applications, such as a conveyer to transport soft materials, a formation process for ordered structures of micro- and/or nanomaterials, a self-cleaning surface and so on.

\section{Control of chemical wave propagation through gap junction in a self-oscillating gel array}

A chemomechanical actuator using a reaction-diffusion wave across a gap junction was constructed toward a novel microconveyor by a micropatterned self-oscillating gel array. ${ }^{31}$ The unidirectional propagation of the chemical wave of the BZ reaction was induced on gel arrays. In the case of using a triangle-shaped gel as an element of the array, the chemical wave propagated from the corner of the triangle gel to the plane side of the other gel (C-to-P) across the gap junction, whereas it propagated from the plane side to the corner side (P-to-C) in the case of the pentagonal gel array. Numerical analysis based on a theoretical model was carried out for understanding the mechanism of unidirectional propagation in triangle and pentagonal gel arrays. By fabricating different shapes of gel arrays, it is possible to control direction. The swelling and deswelling changes of gels followed the unidirectional propagation of the chemical wave.

\section{SELF-OSCILLATING POLYMER CHAINS AND MICROGELS AS 'NANOOSCILLATOR'}

Self-oscillation of polymer chains with rhythmic soluble-insoluble changes

In self-oscillating gel, redox changes of the $\mathrm{Ru}(\mathrm{bpy})_{3}{ }^{2+}$ catalyst are converted to conformational changes of polymer chain by polymerization. The conformational changes are amplified to macroscopic 


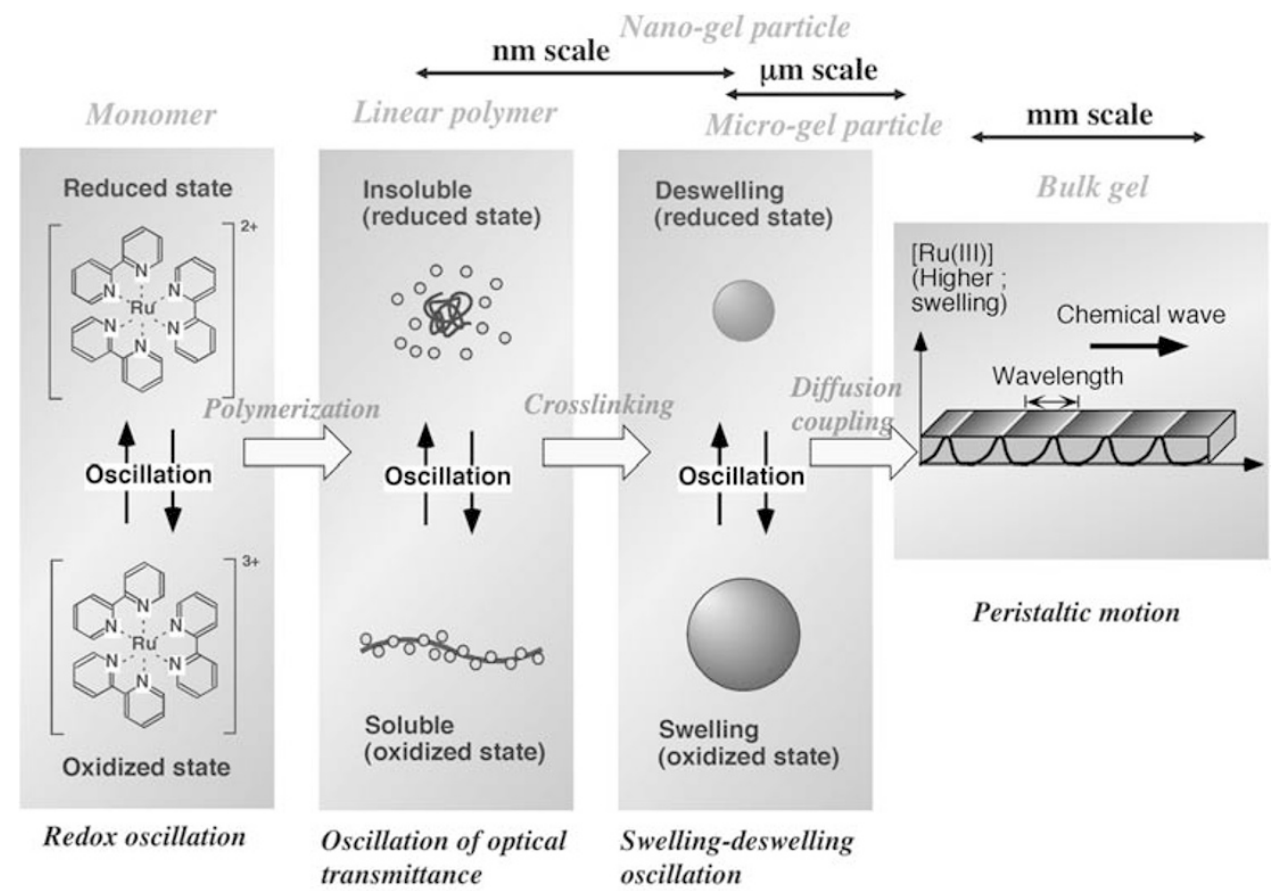

Figure 8 Synchronization in self-oscillating gel from microscopic to macroscopic level. A full color version of this figure is available at Polymer Journal online.

swelling-deswelling changes of the polymer network by crosslinking. Further, when the gel size is larger than the chemical wavelength, the chemical wave propagates in the gel by coupling with diffusion. Thereafter, the peristaltic motion of the gel is created. In this manner, a synchronization process exists from the microscopic to the macroscopic level in the self-oscillating gel (Figure 8).

The periodic changes of linear and uncrosslinked polymer chains can be easily observed as cyclic transparent and opaque changes for the polymer solution with color changes due to the redox oscillation of the catalyst. ${ }^{32}$ Figure 9A shows the oscillation profiles of transmittance for a polymer solution that consists of linear poly(NIPAAmco- $\left.\mathrm{Ru}(\text { bpy })_{3}{ }^{2+}\right), \mathrm{MA}, \mathrm{NaBrO}_{3}$ and $\mathrm{HNO}_{3}$ at constant temperatures. The wavelength $(570 \mathrm{~nm})$ at the isosbestic point of reduced and oxidized states was used to detect the optical transmittance changes based on soluble-insoluble changes of the polymer and not on the redox changes of the $\mathrm{Ru}(\mathrm{bpy})_{3}$ moiety. Synchronized with the periodical changes between the $\mathrm{Ru}(\mathrm{II})$ and $\mathrm{Ru}(\mathrm{III})$ states of the $\mathrm{Ru}(\mathrm{bpy})_{3}{ }^{2+}$ site, the polymer becomes hydrophobic and hydrophilic and exhibits cyclic soluble-insoluble changes.

By grafting the polymers or arraying gel beads on the surface of substrates, we have attempted to design self-oscillating surfaces as nanoconveyers. The self-oscillating polymer was covalently immobilized on a glass surface, and self-oscillation was directly observed at the molecular level by AFM. ${ }^{33}$ The self-oscillating polymer with the $\mathrm{N}$-succinimidyl group was immobilized on an aminosilane-coupled glass plate. Although no oscillation was observed in pure water, nanoscale oscillation was observed in an aqueous solution containing BZ substrates. The amplitude was about $10-15 \mathrm{~nm}$, and the period was about $70 \mathrm{~s}$, although some irregular behavior was observed because of a lack of stirring. The amplitude was less than that in solution, as observed by DLS (23.9 and $59.6 \mathrm{~nm}$ ). This smaller amplitude may be because the structure of the immobilized polymer was a loop-traintail: the moving regions were shorter than those of the soluble polymer. Amplitude and frequency were controlled by the concentration of the reactant, as observed in the solution. Here, nanoscale molecular self-oscillation was observed for the first time. The oscillation polymer chain may be used as a component of a nanoclock or nanomachine.

\section{Self-flocculating/dispersing oscillation of microgels}

We then prepared submicron-sized poly(NIPAAm-co-Ru(bpy $)_{3}{ }^{2+}$ ) gel beads by surfactant-free aqueous precipitation polymerization and analyzed the oscillating behavior. ${ }^{34-40}$ Figure $9 \mathrm{~B}$ shows the oscillation profiles of transmittance for the microgel dispersions. At low temperatures $\left(20-26.5^{\circ} \mathrm{C}\right)$, on raising the temperature, the amplitude of the oscillation became larger. The increase in amplitude is due to the increased deviation of the hydrodynamic diameter between the $\mathrm{Ru}$ (II) and $\mathrm{Ru}(\mathrm{III})$ states. Furthermore, a remarkable change in waveform was observed between 26.5 and $27^{\circ} \mathrm{C}$. The amplitude of the oscillations then dramatically decreased at $27.5^{\circ} \mathrm{C}$, and finally the periodic transmittance changes could no longer be observed at $28^{\circ} \mathrm{C}$. The sudden change in the oscillation waveform should be related to the difference in colloidal stability between the $\mathrm{Ru}$ (II) and $\mathrm{Ru}$ (III) states. Here, the microgels should be flocculated because of a lack of electrostatic repulsion when the microgels were deswollen (see Figure 2). The remarkable change in waveform was only observed at higher dispersion concentrations ( $>0.225 \mathrm{wt} \%)$. The self-oscillating property makes microgels more attractive for future developments, such as the assembly of microgels and optical and rheological applications.

\section{Viscosity oscillation of polymer solution and microgel dispersion} In the case of the self-oscillating polymer solution or the microgel dispersion, solubility changes or the swelling-deswelling changes can be measured as viscosity changes and as optical transmittance changes of the solution or dispersion. In fact, we succeeded in observing the viscosity self-oscillation for the polymer solution induced by the BZ reaction at constant temperature. ${ }^{41}$ The self-oscillation in viscosity was originated by the difference between the solubilities of the polymer 
A
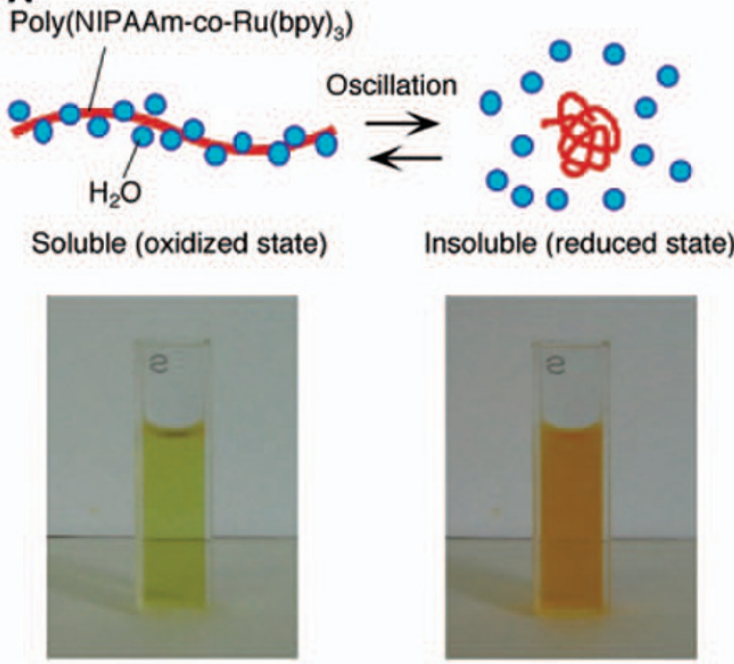

B
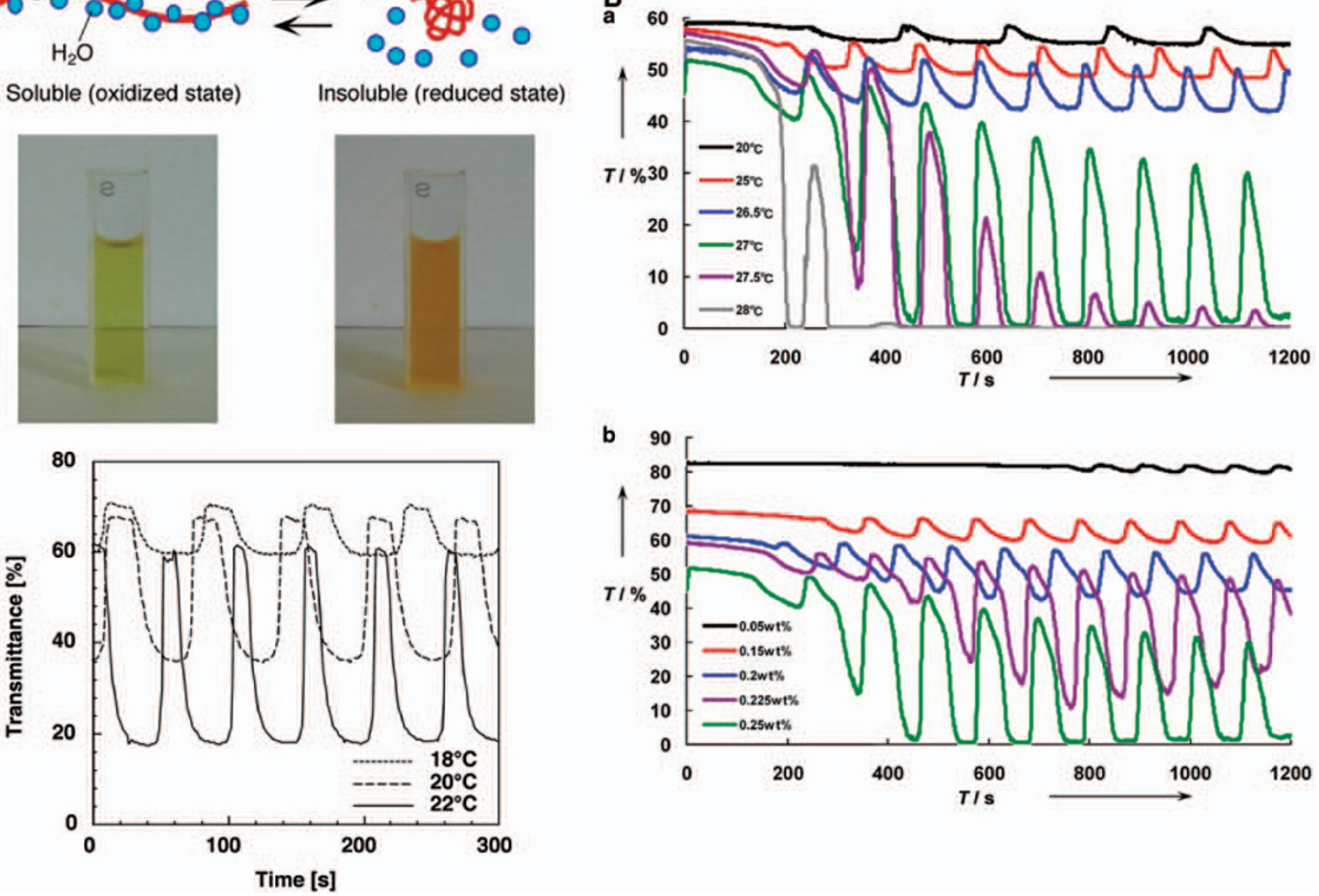

Figure 9 (A) Oscillating profiles of optical transmittance for poly(NIPAAm-co-Ru(bpy) ${ }_{3}^{2+}$ ) solution at several temperatures (Yoshida et al. ${ }^{32}$ ). (B) Selfoscillating profiles of optical transmittance for microgel dispersions. Microgels were dispersed in aqueous solutions containing $\mathrm{MA}(62.5 \mathrm{~mm}), \mathrm{NaBrO}_{3}$ $(84 \mathrm{~mm})$ and $\mathrm{HNO}_{3}(0.3 \mathrm{~m})$. (Upper) The microgel concentration was $0.25 \mathrm{wt} \%$. Profiles measured at different temperatures. (Lower) Profiles measured at different microgel dispersion concentrations at $27^{\circ} \mathrm{C}$ (Suzuki et al. ${ }^{35}$ ).

chain in the reduced and oxidized states. The effects of the polymer concentration and the temperature of the viscosity self-oscillation were investigated. As a result, the self-oscillation in the viscosity significantly depended on the concentration of the polymer and on the temperature of the polymer solution. The period of self-oscillation of the viscosity decreased with increasing temperature in accordance with the Arrhenius equation.

We have recently achieved autonomously oscillating viscosity in a microgel dispersion using autonomously oscillating microgels. ${ }^{39,40} \mathrm{We}$ found that viscosity oscillation occurs in two different ways, exhibiting a simple pulsatile waveform or a complex waveform with two peaks per period (Figure 10a). It was suggested that the difference in waveform is due to the difference in the oscillating manner of the microgels, swelling/deswelling or dispersing/flocculating oscillation, as mentioned before. We can control the rhythm and amplitude of the oscillation using these two phenomena of the microgels. To characterize the viscosity oscillation, two types of microgels were synthesized by changing the feed ratio of $\mathrm{Ru}(\mathrm{bpy})_{3}$ and the crosslinker. The viscosity of the microgel dispersions at high salt concentrations could be controlled by changing the concentrations of the microgels. The autonomously oscillating viscosity was only measured when the concentration of microgels was high. The amplitude of oscillation became larger with increasing concentrations of microgels. By adjusting the concentration of substrates for the BZ reaction, we could achieve constant oscillation for a long time. Moreover, with increasing $\mathrm{Ru}(\mathrm{bpy})_{3}$ and a decrease in the crosslinker, microgels showed a high degree of swelling/deswelling oscillation, resulting in larger amplitudes of autonomously oscillating viscosity (Figure 10b).

This technology could be applied in many applications, as electroor magnetic-rheological fluids have been. In particular, the dispersion with autonomously oscillating viscosity may be used as a micropump, which could realize novel microfluidic devices.

\section{Fabrication of microgel beads monolayer}

As discussed in the previous section, we have been interested in the construction of micro/nanoconveyers by grafting or arraying selfoscillating polymers or gel beads. For this purpose, a fabrication method for organized monolayers of microgel beads was investigated. ${ }^{42}$ A two-dimensional closely packed array of thermosensitive microgel beads was prepared by double-template polymerization. First, a two-dimensional colloidal crystal of silica beads of $10 \mu \mathrm{m}$ diameter was obtained by solvent evaporation. This monolayer of 


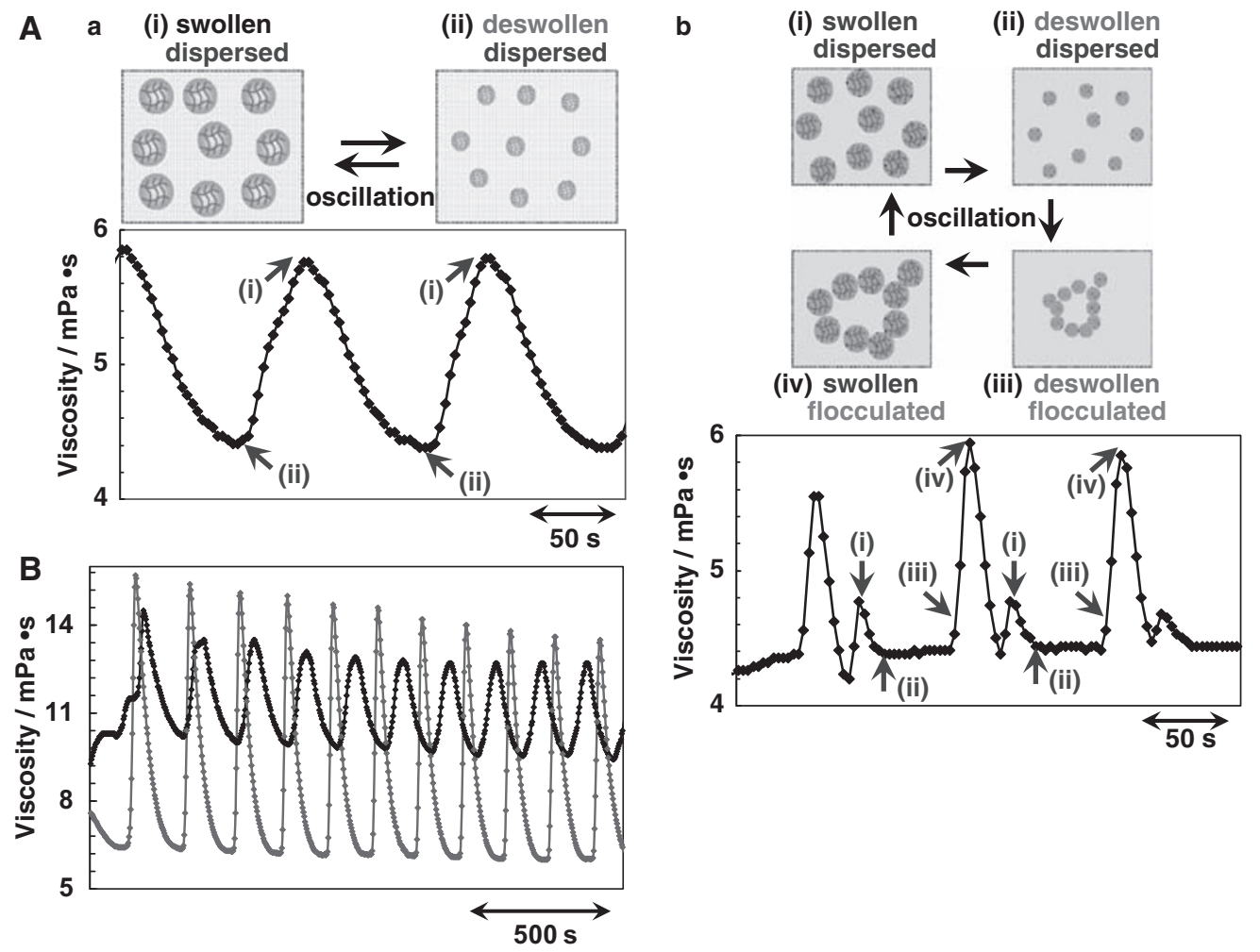

Figure 10 (A) Two different types of oscillating waveforms observed at 20 and $23^{\circ} \mathrm{C}$. The numbers in each oscillating profiles refer to the corresponding illustrations (Suzuki et al. ${ }^{39}$ ). (B) Autonomously oscillating profiles of viscosity in the microgel dispersions measured at $15^{\circ} \mathrm{C}$ for

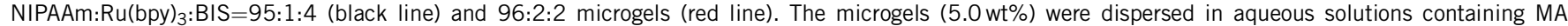
$(100 \mathrm{~mm}), \mathrm{NaBrO}_{3}(150 \mathrm{~mm})$ and $\mathrm{HNO}_{3}(500 \mathrm{~mm})$. Both data were taken at a constant shear rate of $132 \mathrm{~s}^{-1}$ (Suzuki et al. ${ }^{39}$ ). A full color version of this figure is available at Polymer Journal online.

colloidal crystal can serve as the first template for preparation of macroporous polystyrene. The macroporous polystyrene trapping the crystalline order can be used as a negative template for fabricating a gel bead array. Through double-template polymerization, functional surfaces using thermosensitive PNIPAAm gel beads were fabricated. It was observed that the topography of the surface changed with temperature. The fabrication method demonstrated here was so versatile that any kind of gel bead could be obtained. This method may be a key technology used to create a new functional surface. In fact, a monolayer of self-oscillating microgel beads was fabricated by this method, and the chemical wave propagation on the monolayer was observed.

\section{ATTEMPTS OF SELF-OSCILLATION UNDER PHYSIOLOGICAL CONDITIONS}

Self-oscillation of polymer chains under acid-free conditions

Thus far, the author has succeeded in developing a novel selfoscillating polymer (or gel) by utilizing the BZ reaction. However, the operating conditions for the self-oscillation are limited to conditions under which the BZ reaction occurs. For practical applications as functional bio- or biomimetic materials, it is necessary to design a selfoscillating polymer that functions in biological environments. To cause the self-oscillation of polymer systems under physiological conditions, BZ substrates other than organic ones, such as MA and citric acid, must be built into the polymer system itself. Therefore, we undertook two steps. The first step is to design a novel self-oscillating polymer chain with incorporated $\mathrm{pH}$-control sites, that is, polymer chains that exhibit rhythmic oscillations in aqueous solutions containing only the two BZ substrates without using acid as an added agent. For this purpose, AMPS was incorporated into the poly(NIPAAmco- $\mathrm{Ru}(\mathrm{bpy})_{3}{ }^{2+}$ ) chain as the $\mathrm{pH}$-control site. ${ }^{43,44}$

The self-oscillating transmittance change for the solutions of poly(NIPAAm-co-Ru(bpy) ${ }_{3}{ }^{2+}$-co-AMPS $)(20: 10: 70 \mathrm{wt} \%$ in feed $)$ at three constant temperatures $\left(18,21\right.$ and $\left.24^{\circ} \mathrm{C}\right)$ was demonstrated. Under acid-free conditions and in the presence of only two BZ substrates (MA and sodium bromate), we succeeded in causing the soluble-insoluble self-oscillation of a polymer solution. The oscillating behavior was remarkably influenced by the temperature, polymer concentration and composition.

\section{Self-oscillation under oxidant-free conditions}

For the second step, we attempted to introduce the oxidizing agent into the polymer. Methacrylamidopropyltrimethylammonium chloride (MAPTAC), with a positively charged group, was incorporated into poly(NIPAAm-co-Ru(bpy $)_{3}{ }^{2+}$ ) as a capture site for an anionic oxidizing agent (bromate ion). ${ }^{45}$ The bromate ion was introduced into the MAPTAC-containing polymer through ion exchange. Under the conditions in which only two BZ substrates (MA and sulfuric acid) were present, the soluble-insoluble self-oscillation of the polymer was observed.

In the self-oscillating polymer solution system induced by the BZ reaction, self-oscillation was achieved without the addition of an oxidizing agent by using the MAPTAC-containing polymer, which included sodium bromate as a counter ion. The self-oscillating behavior was controllable by temperature. The polymer has two advantages because of its higher LCST: one is self-oscillation around 

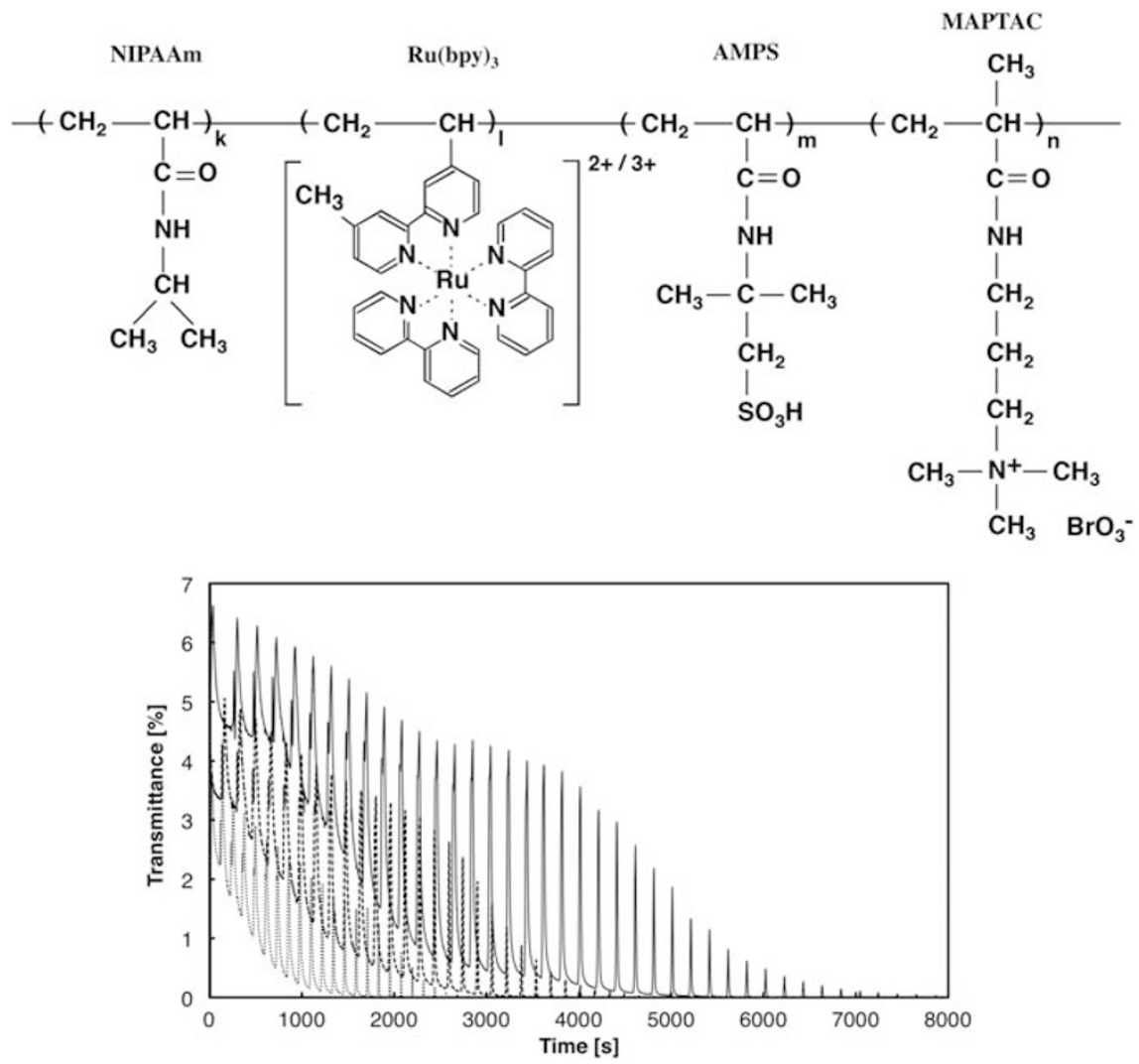

Figure 11 Chemical structure of poly(NIPAAm-co-Ru(bpy) ${ }_{3}^{2+}$-co-AMPS-co-MAPTAC) (upper) and the oscillating profiles of the optical transmittance for the polymer solution at $12^{\circ} \mathrm{C}$ when only MA $(0.7 \mathrm{~m}$ : fine dotted line, $0.5 \mathrm{~m}$ : rough dotted line, $0.3 \mathrm{~m}$ : solid line) is added to the solution (lower) (Hara and Yoshida ${ }^{46}$ ).

body temperature, and the other is oscillation for a longer time without intermolecular aggregation among the polymer chains in the reduced state.

\section{Self-oscillation under acid- and oxidant-free conditions}

Further, we have synthesized a quarternary copolymer that includes both $\mathrm{pH}$-control and oxidant-supplying sites in the poly(NIPAAmco-Ru(bpy $)_{3}$ ) chain at the same time. ${ }^{46}$ In the polymer, AMPS was incorporated as a $\mathrm{pH}$-control site, and MAPTAC with a positively charged group was incorporated as a capture site for an anionic oxidizing agent (bromate ion). Using the polymer, self-oscillation under biological conditions in which only the organic acid (MA) exists was actually achieved (Figure 11). Other than MA, citric acid or malic acid, which is biorelated organic acid, can be a substrate for the BZ reaction. For practical medical use, however, it would be necessary to avoid any exchange of toxic ions such as bromate to the outside of the gel. In addition, the working temperature must be improved. The design of gel for biomedical applications is under investigation.

\section{ACKNOWLEDGEMENTS}

I gratefully thank all my students in the laboratory and the collaborators on this research project, especially the previous postdoc and $\mathrm{PhD}$ students: Dr Daisuke Suzuki (Shinshu University), Dr Takamasa Sakai (University of Tokyo), Dr Yusuke Hara (AIST, Japan), Dr Yasuhiro Maeda (NIMS, Japan), Dr Kosuke Okeyoshi (University of Tokyo) and Dr Yoko Murase (Dai Nippon Printing Co, Ltd, Japan).
1 Yoshida, R. Design of functional polymer gels and their application to biomimetic materials,. Curr. Org. Chem. 9, 1617-1641 (2005).

2 Ottenbrite, R. M., Park, K., Okano, T. \& Peppas, N. A. (eds) Biomedical Applications of Hydrogels Handbook (Springer, New York, 2010).

3 Miyata, T. Stimuli-responsive polymer and gels in Supramolecular Design for Biological Applications (ed. Yui, N.) 191-225 (CRC Press, Boca Raton, 2002).

4 Osada, Y. \& Khokhlov, A. R. (eds) Polymer Gels and Networks (Marcel Dekker, New York, 2002).

5 Field, R. J. \& Burger, M. (eds). Oscillations and Traveling Waves in Chemical Systems (John Wiley \& Sons, New York, 1985).

6 Epstein, I. R. \& Pojman, J. A. An Introduction to Nonlinear Chemical Dynamics: Oscillations, Waves, Patterns, and Chaos (Oxford University Press, New York, 1998).

7 Yoshida, R., Takahashi, T., Yamaguchi, T. \& Ichijo, H. Self-oscillating gel. J. Am. Chem. Soc. 118, 5134-5135 (1996).

8 Yoshida, R., Takahashi, T., Yamaguchi, T. \& Ichijo, H. Self-oscillating gels. Adv. Mater. 9, 175-178 (1997).

9 Yoshida, R. Self-oscillating polymer and gels as novel biomimetic materials. Bull. Chem. Soc. Jpn 81, 676-688 (2008).

10 Yoshida, R., Sakai, T., Hara, Y., Maeda, S., Hashimoto, S., Suzuki, D. \& Murase, Y. Selfoscillating gel as novel biomimetic materials. J. Controlled Release 140, 186-193 (2009).

11 Yoshida, R. Self-oscillating gels driven by the Belouzov-Zhabotinsky reaction as novel smart materials. Adv. Mater. 22, 3463-3483 (2010).

12 Yoshida, R., Tanaka, M., Onodera, S., Yamaguchi, T. \& Kokufuta, E. In-phase synchronization of chemical and mechanical oscillations in self-oscillating gels,. J. Phys. Chem. A 104, 7549-7555 (2000).

13 Yoshida, R., Takei, K. \& Yamaguchi, T. Self-beating motion of gels and modulation of oscillation rhythm synchronized with organic acid,. Macromolecules 36, 1759-1761 (2003).

14 Ito, Y., Nogawa, N. \& Yoshida, R. Temperature control of the Belousov-Zhabotinsky reaction using a thermo-responsive polymer. Langmuir 19, 9577-9579 (2003).

15 Yoshida, R., Sakai, T., Tabata, O. \& Yamaguchi, T. Design of novel biomimetic polymer gels with self-oscillating function. Sci. Tech. Adv. Mater. 3, 95-102 (2002).

16 Takeoka, Y., Watanabe, M. \& Yoshida, R. Self-sustaining peristaltic motion on the surface of a porous gel. J. Am. Chem. Soc. 125, 13320-13321 (2003). 
17 Shinohara, S., Seki, T., Sakai, T., Yoshida, R. \& Takeoka, Y. Chemical and optical control of peristaltic actuator based on self-oscillating porous gel. Chem. Commun. 4735-4737 (2008).

18 Shinohara, S., Seki, T., Sakai, T., Yoshida, R. \& Takeoka, Y. Photoregulated wormlike motion of a gel. Angew. Chem. Int. Ed. 47, 9039-9043 (2008).

19 Maeda, S., Hara, Y., Yoshida, R. \& Hashimoto, S. Peristaltic motion of polymer gels. Angew. Chem. Int. Ed. 47, 6690-6693 (2008).

20 Sasaki, S., Koga, S., Yoshida, R. \& Yamaguchi, T. Mechanical oscillation coupled with the Belousov-Zhabotinsky reaction in gel. Langmuir 19, 5595-5600 (2003).

21 Aoki, R., Enoki, M. \& Yoshida, R. Mechanical behavior during self-oscillating of NIPAAm-co-Ru(bpy) ${ }_{3}$ gel. Key Eng. Mater. 353, 2235-2238 (2007).

22 Yoshida, R., Omata, K., Yamaura, K., Ebata, M., Tanaka, M. \& Takai, M. Maskless microfabrication of thermosensitive gels using a microscope and application to a controlled release microchip. Lab Chip 6, 1384-1386 (2006).

23 Yoshida, R., Omata, K., Yamaura, K., Sakai, T., Hara, Y., Maeda, S. \& Hashimoto, S. Microfabrication of functional polymer gels and their application to novel biomimetic materials. J. Photopolymer Sci. Tech. 19, 441-444 (2006).

24 Tabata, O., Hirasawa, H., Aoki, S., Yoshida, R. \& Kokufuta, E. Ciliary motion actuator using self-oscillating gel. Sens. Actuators A 95, 234-238 (2002).

25 Tabata, O., Kojima, H., Kasatani, T., Isono, Y. \& Yoshida, R. Chemo-mechanical actuator using self-oscillating gel for artificial cilia. Proceedings of the International Conference on MEMS 2003 pp. 12-15 (2003).

26 Maeda, S., Hara, Y., Sakai, T., Yoshida, R. \& Hashimoto, S. Self-walking gel. Adv. Mater. 19, 3480-3484 (2007).

27 Maeda, S., Hara, Y., Yoshida, R. \& Hashimoto, S. Control of dynamic motion of a gel actuator driven by the Belousov-Zhabotinsky reaction. Macromol. Rapid Commun. 29, 401-405 (2008).

28 Murase, Y., Maeda, S., Hashimoto, S. \& Yoshida, R. Design of a mass transport surface utilizing peristaltic motion of a self-oscillating gel. Langmuir 25, 483-489 (2009).

29 Murase, Y., Takeshima, R. \& Yoshida, R. Design of mass transport surface using selfoscillating gel. Trans. Mater. Res. Soc. Japan 34, 171-174 (2009).

30 Murase, Y., Hidaka, M. \& Yoshida, R. Self-driven gel conveyer: autonomous transportation by peristaltic motion of self-oscillating gel. Sens. Actuators B 149, 272-283 (2010).

31 Tateyama, S., Shibuta, Y. \& Yoshida, R. Direction control of chemical wave propagation in self-oscillating gel array. J. Phys. Chem. B 112, 1777-1782 (2008).
32 Yoshida, R., Sakai, T., Ito, S. \& Yamaguchi, T. Self-oscillation of polymer chains with rhythmical soluble-insoluble changes. J. Am. Chem. Soc. 124, 8095-8098 (2002).

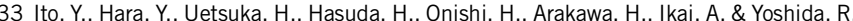
AFM observation of immobilized self-oscillating polymer. J. Phys. Chem. B 110, 5170-5173 (2006).

34 Sakai, T. \& Yoshida, R. Self-oscillating nanogel particles. Langmuir 20, 1036-1038 (2004).

35 Suzuki, D., Sakai, T. \& Yoshida, R. Self-flocculating/self-dispersing oscillation of microgels. Angew. Chem. Int. Ed. 47, 917-920 (2008).

36 Suzuki, D. \& Yoshida, R. Temporal control of self-oscillation for microgels by crosslinking network structure. Macromolecules 41, 5830-5838 (2008).

37 Suzuki, D. \& Yoshida, R. Effect of initial substrate concentration of the BelousovZhabotinsky reaction on self-oscillation for microgel system. J. Phys. Chem. B 112, 12618-12624 (2008).

38 Suzuki, D. \& Yoshida, R. Self-oscillating core/shell microgels: effect of a crosslinked nanoshell on autonomous oscillation of the core. Polym. J. 42, 501-508 (2010).

39 Suzuki, D., Taniguchi, H. \& Yoshida, R. Autonomously oscillating viscosity in microgel dispersions. J. Am. Chem. Soc. 131, 12058-12059 (2009).

40 Taniguchi, H., Suzuki, D. \& Yoshida, R. Characterization of autonomously oscillating viscosity induced by swelling/deswelling oscillation of the microgels. J. Phys. Chem. B $114,2405-2410$ (2010)

41 Hara, Y. \& Yoshida, R. A viscosity self-oscillation of polymer solution induced by the BZ reaction under acid-free condition. J. Chem. Phys. 128, 224904 (2008).

42 Sakai, T., Takeoka, Y., Seki, T. \& Yoshida, R. Organized monolayer of thermosensitive microgel beads prepared by double-templete polymerization. Langmuir 23, 8651-8654 (2007).

43 Hara, Y. \& Yoshida, R. Self-oscillation of polymer chains induced by the BelousovZhabotinsky reaction under acid-free conditions. J. Phys. Chem. B 109, 9451-9454 (2005).

44 Hara, Y. \& Yoshida, R. Control of oscillating behavior for the self-oscillating polymer with $\mathrm{pH}$-control site,. Langmuir 21, 9773-9776 (2005).

45 Hara, Y., Sakai, T., Maeda, S., Hashimoto, S. \& Yoshida, R. Self-oscillating solubleinsoluble changes of polymer chain including an oxidizing agent induced by the Belousov-Zhabotinsky reaction. J. Phys. Chem. B 109, 23316 (2005).

46 Hara, Y. \& Yoshida, R. Self-oscillating polymer fueled by organic acid. J. Phys. Chem. B $112,8427-8429$ (2008)

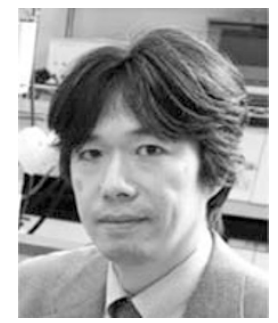

Ryo Yoshida received his $\mathrm{PhD}$ in Chemical Engineering from Waseda University, Japan, in 1993. He was a Research Associate at Tokyo Women's Medical University in 1993, a Researcher at the National Institute of Materials and Chemical Research in 1994-1997 and Assistant Professor at the University of Tsukuba in 1997-2000. In 2001, he moved to the University of Tokyo as Associate Professor. He won the SPSJ (the Society of Polymer Science, Japan) Wiley Award in 2009. 